\title{
Lower Intraprocedural Systolic Blood Pressure Predicts Good Outcome in Patients Undergoing Endovascular Therapy for Acute Ischemic Stroke
}

\author{
Seby John ${ }^{a}$ Walaa Hazaa ${ }^{a}$ Ken Uchino ${ }^{a}$ Gabor Toth ${ }^{a}$ Mark Bain ${ }^{a}$ \\ Umera Thebo $^{\mathrm{b}}$ Muhammad S. Hussain ${ }^{a}$ \\ ${ }^{a}$ Cerebrovascular Center and ${ }^{\mathrm{b}}$ Department of Internal Medicine, Cleveland Clinic, \\ Cleveland, Ohio, USA
}

\section{Key Words}

Acute ischemic stroke $\cdot$ Intra-arterial therapy $\cdot$ Hemodynamics $\cdot$ Blood pressure $\cdot$ Maximum systolic blood pressure - Maximum mean arterial pressure · Hypertension · Outcome

\begin{abstract}
Background: It is unknown if intraprocedural blood pressure (BP) influences clinical outcomes and what BP parameter best predicts outcomes in acute ischemic stroke (AIS) patients who undergo intra-arterial therapy (IAT) for emergent large vessel occlusion. Methods: We retrospectively reviewed 147 patients who underwent IAT for anterior circulation AIS from January 2008 to December 2012 at our institution. Baseline demographics, stroke treatment variables, and detailed intraprocedural hemodynamic variables were collected. Results: The entire cohort consisted of 81 (55\%) females with a mean age of $66.9 \pm 15.6$ years and a median National Institutes of Health Stroke Scale (NIHSS) score of 16 (IQR 11-21). Thirty-six (24.5\%) patients died during hospitalization, 25 (17\%) achieved a 30-day modified Rankin Scale score of $0-2$, and 24 (16.3\%) suffered symptomatic parenchymal hematoma type 1/2 hemorrhage. Patients who achieved a good outcome had a significantly lower admission NIHSS score, a higher baseline CT ASPECTS score, and a lower rate of ICA terminus occlusions. Successful recanalization was more frequent in the good-outcome group, while symptomatic hemorrhages occurred only in poor-outcome patients. The first systolic BP (SBP; $146.5 \pm 0.2$ vs. $157.7 \pm 25.6$ $\mathrm{mm} \mathrm{Hg}, \mathrm{p}=0.042$ ), first mean arterial pressure (MAP; $98.1 \pm 20.8$ vs. $109.7 \pm 20.3 \mathrm{~mm} \mathrm{Hg}, \mathrm{p}=$ 0.024), maximum SBP (164.6 \pm 27.6 vs. $180.9 \pm 18.3 \mathrm{~mm} \mathrm{Hg}, \mathrm{p}=0.0003)$, and maximum MAP (125.5 \pm 18.6 vs. $138.5 \pm 24.6 \mathrm{~mm} \mathrm{Hg}, \mathrm{p}=0.0309)$ were all significantly lower in patients who achieved good outcomes. A lower maximum intraprocedural SBP was an independent predictor of good outcome (adjusted OR 0.929, 95\% CI 0.886-0.963, p = 0.0005). Initial NIHSS score
\end{abstract}


was the only other independent predictor of a good outcome. Conclusion: Lower intraprocedural SBP was associated with good outcome in patients undergoing IAT for AIS, and maximum SBP was an independent predictor of good outcome. SBP may be the optimal hemodynamic variable to monitor intraprocedurally during IAT and may predict outcome.

(C) 2016 S. Karger AG, Basel

\section{Introduction}

There are many factors that influence outcomes of patients with acute ischemic stroke (AIS) from emergent large vessel occlusion who undergo intra-arterial recanalization therapy (IAT). Age, initial stroke severity, and time from symptom onset to vessel recanalization are few variables that have been well characterized [1]. Arterial blood pressure (BP) is a dynamic variable that is often altered in AIS, and multiple studies have investigated the relationship between various BP parameters during admission for AIS and clinical outcomes. BP is largely a surrogate for cerebral perfusion pressure [2]. Extreme arterial hypertension or hypotension is clearly deleterious to multiple organ systems. However, moderate hypertension during AIS would seem theoretically advantageous since it would improve cerebral perfusion to the ischemic tissue, while at the same time carrying the risk of worsening cerebral edema and promoting hemorrhagic transformation. As such, there is likely a BP range that is optimal for maintaining cerebral perfusion for each individual during an ischemic stroke. However, there is limited scientific evidence for such a target range.

For patients who undergo IAT for AIS, the 2013 American Stroke Association guidelines recommend maintaining the BP at or below $180 / 105 \mathrm{~mm} \mathrm{Hg}$, similar to that recommended after intravenous (IV) thrombolysis using recombinant tissue plasminogen activator (tPA) [3]. However, it is unknown if intraprocedural BP influences clinical outcomes and what BP parameter best predicts outcomes. Knowledge of this would help with the development of more specific intraprocedural BP parameters. In this retrospective study, we sought to explore the influence of intraprocedural hemodynamic parameters on outcomes of AIS patients undergoing IAT.

\section{Methods}

Subjects

This study was conducted after Institutional Review Board approval. Our institution's prospectively maintained database of AIS interventions was retrospectively reviewed to identify all patients who underwent IAT for AIS between January 2008 and December 2012. Subjects 18 years of age and older with acute anterior circulation ischemic stroke who underwent IAT were included in the present analysis. Posterior circulation strokes were excluded due to the higher likelihood of death or severe disability. The decision for IAT was made based on previously published protocols [4] and included only patients with emergent large vessel occlusion.

\section{Demographics and Time Points}

Demographic data including age, gender, and vascular risk factors; National Institutes of Health Stroke Scale (NIHSS) scores on admission and IV tPA use, and anesthesia type were collected. For elapsed time points, we collected last know well time, time to tPA administration, groin incision time, time to first angiographic run, time to reach target vessels, and time to recanalization. Discharged patients were followed up at around 30 days from the time of discharge to assess functional status, either by out-patient stroke clinic evaluation or via telephone call by a stroke nurse or by review of medical records by a stroke fellow if sufficient documentation was present. The modified Rankin Scale (mRS) score was recorded for these patients. All individuals were mRS certified. In-hospital mortality was also captured. 
Hemodynamic and Radiographic Variables

Hemodynamic variables collected from the anesthesia records included heart rate, systolic BP (SBP), diastolic BP (DBP), and mean arterial pressure (MAP) right before the start of the case; maximum and minimum intraprocedural SBP, DBP, MAP, and heart rate; episodes of intraprocedural SBP <90 mm Hg; episodes of intraprocedural SBP $<120 \mathrm{~mm} \mathrm{Hg}$; episodes of intraprocedural SBP drop $>20 \%$ of baseline SBP, and episodes of intraprocedural MAP $<60 \mathrm{~mm} \mathrm{Hg}$. All patients undergoing IAT got an arterial line, and BP was recorded at 5-min intervals by the Anesthesia Record Keeping System.

Radiographic variables recorded included site of vessel occlusion, recanalization grade, and postprocedural intracerebral hemorrhage. Recanalization was graded using the Thrombolysis in Cerebral Infarction (TICI) score, and successful recanalization was defined as TICI grade 2b-3 [5]. Hemorrhages were classified as parenchymal hematoma or hemorrhagic infarction, based on previously published criteria [6]. Hemorrhages were reviewed on noncontrast head CT images obtained $24 \mathrm{~h}$ after the procedure.

\section{Outcomes}

We assessed outcomes using the mRS, dichotomized to $\leq 2$ to define favorable functional outcome and to $>2$ for unfavorable outcome.

\section{Statistical Analysis}

Statistical analysis was performed using JMP version 10. Univariate analysis of categorical variables was performed with Fisher's exact test and Student's t test for continuous variables in comparing patients who achieved good versus poor outcomes after IAT. A multivariable logistic regression analysis was constructed with variables to determine independent predictors of good outcome, mortality, and symptomatic hemorrhage. A p value of $<0.05$ was considered statistically significant

\section{Results}

A total of 213 subjects underwent IAT for AIS during the prespecified study period, and 147 patients ultimately met inclusion criteria for the present investigation. Twenty-three patients were excluded due to IAT performed for posterior circulation vessel occlusion, and 30-day mRS outcomes were not available in 43 patients. The entire cohort consisted of 81 $(55 \%)$ females with a mean age of $66.9 \pm 15.6$ years and a median NIHSS score of 16 (IQR 11-21). The median admission CT ASPECTS score was 8 (IQR 6-9), and 52 (35.8\%) patients received IV tPA. Sixty-eight (46\%) patients underwent IAT under general anesthesia, 74 (50\%) patients achieved TICI $2 \mathrm{~b}-3$ recanalization, and 24 (16.3\%) patients suffered symptomatic parenchymal hematoma type $1 / 2$ hemorrhage. Thirty-six $(24.5 \%)$ patients died during the hospitalization, and 25 (17\%) achieved a 30-day good outcome of an mRS score of $0-2$.

Table 1 summarizes the univariate analysis comparing predictors of outcome after endovascular therapy for acute stroke. Patients who achieved a good outcome had a significantly lower admission NIHSS score (11.7 \pm 6.2 vs. $16.6 \pm 6.5, \mathrm{p}=0.0007)$, a higher baseline CT ASPECTS score ( $8.6 \pm 1.8$ vs. $7.6 \pm 1.8, \mathrm{p}=0.015$ ), and had lesser ICA terminus occlusions ( 8 vs. $31.9 \%, p=0.007)$. Successful recanalization was more frequent in the good-outcome group ( 80 vs. $44.2 \%, \mathrm{p}=0.0008$ ), while symptomatic hemorrhages were more frequent in the poor-outcome group ( 0 vs. $19.7 \%$, p = 0.0017).

Table 2 compares the intraprocedural hemodynamic variables between the two groups. The first SBP ( $146.5 \pm 20.2$ vs. $157.7 \pm 25.6 \mathrm{~mm} \mathrm{Hg}, \mathrm{p}=0.042)$, first MAP ( $98.1 \pm 20.8$ vs. 109.7 $\pm 20.3 \mathrm{~mm} \mathrm{Hg}, \mathrm{p}=0.024)$, maximum SBP (164.6 \pm 27.6 vs. $180.9 \pm 18.3 \mathrm{~mm} \mathrm{Hg}, \mathrm{p}=0.0003)$, and maximum MAP (125.5 \pm 18.6 vs. $138.5 \pm 24.6 \mathrm{~mm} \mathrm{Hg}, \mathrm{p}=0.0309)$ were all significantly lower in patients who achieved good outcomes. There were no differences in any of the other hemodynamic variables studied between the two groups. SBP variability calculated by the difference between the maximum and minimum SBP was also similar in both groups. 
Table 1. Baseline characteristics

\begin{tabular}{lllll}
\hline Variable & $\begin{array}{l}\text { Total } \\
(\mathrm{n}=147)\end{array}$ & $\begin{array}{l}\mathrm{mRS} 0-2 \\
(\mathrm{n}=25)\end{array}$ & $\begin{array}{l}\mathrm{mRS} 3-6 \\
(\mathrm{n}=122)\end{array}$ & $\mathrm{p}$ value \\
\hline Age, years & $67 \pm 16$ & $65 \pm 14$ & $67 \pm 16$ & 0.447 \\
Female & $81(55.1)$ & $10(40)$ & $71(58)$ & 0.096 \\
Baseline NIHSS score & $15.8 \pm 6.7$ & $11.7 \pm 6.2$ & $16.6 \pm 6.5$ & 0.0007 \\
Baseline CT ASPECTS score & $7.7 \pm 1.8$ & $8.6 \pm 1.8$ & $7.6 \pm 1.8$ & 0.015 \\
ICA terminus & $41(27.9)$ & $2(8)$ & $39(31.9)$ & 0.007 \\
M1 proximal & $52(35.3)$ & $10(40)$ & $42(34.4)$ & 0.598 \\
M1 distal & $22(14.9)$ & $7(28)$ & $15(12.3)$ & 0.061 \\
M2 & $18(12.2)$ & $3(12)$ & $15(12.3)$ & 0.967 \\
IV tPA & $52(35.8)$ & $9(37.5)$ & $43(35.5)$ & 0.855 \\
Symptom onset to IV tPA, min & $140.9 \pm 56.3$ & $155.1 \pm 55.6$ & $137.5 \pm 56.6$ & 0.405 \\
Symptom onset to recanalization, min & $457.2 \pm 231.7$ & $427.1 \pm 242.7$ & $465.1 \pm 24.5$ & 0.487 \\
TICI 2b-3 & $74(50.3)$ & $20(80)$ & $54(44.2)$ & 0.0008 \\
PH 1 + 2 & $24(16.3)$ & 0 & $24(19.7)$ & 0.0017 \\
\hline
\end{tabular}

Values are mean \pm SD or $\mathrm{n}(\%)$. PH $1+2=$ Parenchymal hematoma type 1 and 2 .

Table 2. Hemodynamic variables based on outcome

\begin{tabular}{lcrl}
\hline Variable & mRS $0-2$ & mRS $3-6$ & p value \\
\hline First SBP, mm Hg & $146.5 \pm 20.2$ & $157.7 \pm 25.6$ & 0.042 \\
First DBP, mm Hg & $77.3 \pm 15.2$ & $79.7 \pm 16.2$ & 0.496 \\
First MAP, mm Hg & $98.1 \pm 20.8$ & $109.7 \pm 20.3$ & 0.024 \\
First HR, bpm & $86.5 \pm 18.2$ & $82.7 \pm 18.9$ & 0.359 \\
Max. SBP, mm Hg & $164.6 \pm 27.6$ & $180.9 \pm 18.3$ & 0.0003 \\
Max. DBP, mm Hg & $89.9 \pm 15.7$ & $98.4 \pm 22.4$ & 0.075 \\
Max. MAP, mm Hg & $125.5 \pm 18.6$ & $138.5 \pm 24.6$ & 0.0309 \\
Max. HR, bpm & $101.3 \pm 21.8$ & $99.1 \pm 21.2$ & 0.642 \\
Min. SBP, mm Hg & $103.1 \pm 23.6$ & $111.5 \pm 23.6$ & 0.109 \\
Min. DBP, mm Hg & $52.3 \pm 15.9$ & $53.1 \pm 13.8$ & 0.799 \\
Min. MAP, mm Hg & $71.8 \pm 16.1$ & $73.3 \pm 15.5$ & 0.709 \\
Min. HR, bpm & $62.3 \pm 20.3$ & $56.4 \pm 16.9$ & 0.123 \\
Patients with SBP drop >20\% from baseline & $17(68)$ & $88(72.3)$ & 0.717 \\
Patients with MAP <60 mm Hg & $5(26.3)$ & $13(12.4)$ & 0.139 \\
\hline
\end{tabular}

Values are mean \pm SD or $\mathrm{n}(\%) . \mathrm{HR}=$ Heart rate; Max. = maximum; Min. = minimum.

Table 3 summarizes the multivariable logistic regression model for independent predictors of a good outcome. A lower maximum intraprocedural SBP was found to be an independent predictor of a good outcome (adjusted OR 0.929, 95\% CI 0.886-0.963, p = $0.0005)$. Initial NIHSS was the only other predictor of a good outcome. A similar analysis was performed to determine predictors of mortality and symptomatic hemorrhage. Increased age, higher initial NIHSS, use of general anesthesia, and development of symptomatic hemorrhage were independent predictors of mortality. The CT ASPECTS score was the only independent predictor of symptomatic hemorrhage. 
John et al.: Lower Intraprocedural Systolic Blood Pressure Predicts Good Outcome in Patients Undergoing Endovascular Therapy for Acute Ischemic Stroke

Table 3. Multivariable logistic regression analysis for good outcomes, mortality, and parenchymal hematoma ${ }^{\mathrm{a}}$

\begin{tabular}{llccc}
\hline Outcome & Variable & Adjusted OR & $95 \%$ CI & p value \\
\hline mRS 0-2 & Initial NIHSS score & 0.827 & $0.694-0.945$ & 0.0137 \\
& Max. SBP & 0.929 & $0.886-0.963$ & 0.0005 \\
\hline Mortality & Age & 1.082 & $1.032-1.150$ & 0.0005 \\
& Initial NIHSS score & 1.127 & $1.024-1.260$ & 0.0132 \\
& General anesthesia & 3.748 & $1.085-15.102$ & 0.0363 \\
& PH 1 + 2 & 11.921 & $2.633-68.319$ & 0.0010 \\
\hline PH 1+2 & CT ASPECTS & 0.637 & $0.454-0.858$ & 0.0048 \\
\hline
\end{tabular}

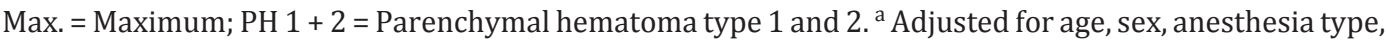
maximum SBP, initial NIHSS score, IV tPA, CT ASPECTS, ICT terminus occlusion, successful recanlization, time to recanalization, and symptomatic hemorrhage.

\section{Discussion}

Our study revealed that AIS patients who underwent IAT and achieved an mRS score of 0-2 had a lower mean maximum SBP compared to those who did not achieve a good outcome. Among all the hemodynamic variables studied, maximum intraprocedural SBP was the only independent predictor of a good outcome. To the best of our knowledge, this is the first report that details the influence of hemodynamic factors on the outcome of AIS patients undergoing IAT.

Multiple studies have investigated the relationship between admission BP parameters in patients with AIS and clinical outcomes [7-10]. These studies have reported a U-shaped relationship between the admission BP and good clinical outcome. Other studies have reported worse clinical outcomes with elevated in-hospital BP, with a more linear association [11-19]. There have been no studies that have examined the influence of intraprocedural hemodynamics on clinical outcome and mortality in patients with AIS who undergo IAT. It is also not clear which parameter among SBP, DBP, or MAP is superior in monitoring patients during the procedure.

A detailed analysis of intraprocedural hemodynamics in our study suggests that SBP may be the correct hemodynamic variable to monitor in these patients. Although no specific recommendations are available regarding intraprocedural BP management, the American Stroke Association guidelines recommend BP targets similar to patients who receive IV thrombolysis, which includes keeping SBP under $180 \mathrm{~mm} \mathrm{Hg}$ and DBP under $105 \mathrm{~mm} \mathrm{Hg}$. Patients in our study who did not achieve good outcomes had a mean maximum intraprocedural SBP of 180.9 (SD \pm 18.3 ) $\mathrm{mm} \mathrm{Hg}$. As such, a lower intraprocedural SBP target such as $160 \mathrm{~mm} \mathrm{Hg}$ like that seen with our patients who achieved an mRS score of 0-2 may be associated with better outcomes and requires further rigorous study.

All 24 patients with symptomatic hemorrhage did not achieve a good outcome. Symptomatic hemorrhage was not an independent predictor of good outcomes but was a strong independent predictor of mortality (adjusted OR 11.921, 95\% CI 2.633-68.319, $\mathrm{p}=0.0010$ ). It is unclear why a higher SBP was associated with worse outcomes in our study. One could speculate about the correlation between higher BP and the increased risk of intracerebral hemorrhage, but this correlation is unknown. In our cohort, the CT ASPECTS score was the only independent predictor of symptomatic hemorrhage. Previous studies such as the European Cooperative Acute Stroke Study I [20], the International Stroke Trial [21], the 
National Institute of Neurological Disorders and Stroke trial [22], or the Interventional Management of Stroke trial [23] did not find an association with BP and hemorrhage. However, intracerebral hemorrhage has been associated with elevated BP after tPA treatment [6, 19, $24]$. Worse outcomes with higher BP may be linked to an increased risk of cerebral edema when there is concurrent high BP $[10,21,25]$.

Our study has multiple limitations most important of which are the retrospective study design that is prone to selection bias and the small sample size. This is a single-center retrospective analysis, and caution should be exercised in generalizing the results obtained in our study. We acknowledge that rates of successful recanalization and good outcomes in this cohort are low compared to recently published positive randomized controlled trials. However, techniques and devices used for IAT during the majority of the time period in our study were drastically different and less effective at achieving recanalization. In addition, baseline disability and late presentation among other variables did not necessarily preclude the selection of patients for IAT. Hemodynamic variables were documented at 5 -min intervals, and it is possible that significant hemodynamic fluctuations were missed in the interval period. Only a single value of the maximum and minimum BP for the whole procedure was finally analyzed, irrespective of recanalization statuses or whether it was before or after recanalization in successfully recanalized patients. However, recanalization status was controlled for in the multivariable logistic regression analysis for all outcomes. Information regarding the use of antihypertensive medications during the procedure was not collected. It is possible that such medications administered in patients with very high BPs could affect outcomes independently.

\section{Conclusion}

Our study found that lower SBP was associated with good outcomes in patients undergoing IAT for AIS and that maximum SBP was an independent predictor of good clinical outcome. SBP may be the optimal hemodynamic variable to monitor intraprocedurally during IAT and may predict outcome. A maximum SBP of $160 \mathrm{~mm} \mathrm{Hg}$ may be a reasonable target to maintain during the procedure, but this needs further study. Larger prospective studies with randomization to different SBP targets are required to see if SBP influences outcomes of AIS patients who undergo IAT and to scientifically determine the optimal BP range during the procedure.

\section{Disclosure Statement}

The authors have no conflicts of interest to disclose.

\section{References}

1 Lee WI, Mitchell P, Dowling R, Yan B: Clinical factors are significant predictors of outcome post intra-arterial therapy for acute ischaemic stroke: a review. J Neuroradiol 2013;40:315-325.

2 Zazulia AR, Videen TO, Powers WJ: Symptomatic autoregulatory failure in acute ischemic stroke. Neurology 2007;68:389-390.

3 Jauch EC, Saver JL, Adams HP Jr, et al; American Heart Association Stroke Council; Council on Cardiovascular Nursing; Council on Peripheral Vascular Disease; Council on Clinical Cardiology: Guidelines for the early management of patients with acute ischemic stroke: a guideline for healthcare professionals from the American Heart Association/American Stroke Association. Stroke 2013;44:870-947.

4 Wisco D, Uchino K, Saqqur M, et al: Addition of hyperacute MRI aids in patient selection, decreasing the use of endovascular stroke therapy. Stroke 2014;45:467-472. 
5 Yoo AJ, Simonsen CZ, Prabhakaran S, et al: Refining angiographic biomarkers of revascularization: improving outcome prediction after intra-arterial therapy. Stroke 2013;44:2509-2512.

6 Larrue V, von Kummer RR, Muller A, Bluhmki E: Risk factors for severe hemorrhagic transformation in ischemic stroke patients treated with recombinant tissue plasminogen activator: a secondary analysis of the European-Australasian Acute Stroke Study (ECASS II). Stroke 2001;32:438-441.

7 Castillo J, Leira R, García MM, Serena J, Blanco M, Dávalos A: Blood pressure decrease during the acute phase of ischemic stroke is associated with brain injury and poor stroke outcome. Stroke 2004;35:520-526.

8 Leonardi-Bee J, Bath PM, Phillips SJ, Sandercock PA; IST Collaborative Group: Blood pressure and clinical outcomes in the International Stroke Trial. Stroke 2002;33:1315-1320.

9 Okumura K, Ohya Y, Maehara A, Wakugami K, Iseki K, Takishita S: Effects of blood pressure levels on case fatality after acute stroke. J Hypertens 2005;23:1217-1223.

10 Vemmos KN, Tsivgoulis G, Spengos K, Zakopoulos N, Synetos A, Manios E, Konstantopoulou P, Mavrikakis M: U-shaped relationship between mortality and admission blood pressure in patients with acute stroke. J Intern Med 2004;255:257-265.

11 Aslanyan S, Fazekas F, Weir CJ, Horner S, Lees KR; GAIN International Steering Committee and Investigators: Effect of blood pressure during the acute period of ischemic stroke on stroke outcome: a tertiary analysis of the GAIN International Trial. Stroke 2003;34:2420-2425.

12 Aslanyan S, Weir CJ, Lees KR; GAIN International Steering Committee and Investigators: Elevated pulse pressure during the acute period of ischemic stroke is associated with poor stroke outcome. Stroke 2004; 35:e153-e155.

13 Boreas AM, Lodder J, Kessels F, de Leeuw PW, Troost J: Prognostic value of blood pressure in acute stroke. J Hum Hypertens 2002;16:111-116.

14 Chamorro A, Vila N, Ascaso C, Elices E, Schonewille W, Blanc R: Blood pressure and functional recovery in acute ischemic stroke. Stroke 1998;29:1850-1853.

15 Grabska K, Niewada M, Sarzynska-Dlugosz I, Kaminski B, Czlonkowska A: Pulse pressure: independent predictor of poor early outcome and mortality following ischemic stroke. Cerebrovasc Dis 2009;27:187-192.

16 Jensen MB, Yoo B, Clarke WR, Davis PH, Adams HR Jr: Blood pressure as an independent prognostic factor in acute ischemic stroke. Can J Neurol Sci 2006;33:34-38.

17 Rodríguez-García JL, Botia E, de La Sierra A, Villanueva MA, González-Spínola J: Significance of elevated blood pressure and its management on the short-term outcome of patients with acute ischemic stroke. Am J Hypertens 2005;18:379-384.

18 Yong M, Diener HC, Kaste M, Mau J: Characteristics of blood pressure profiles as predictors of long-term outcome after acute ischemic stroke. Stroke 2005;36:2619-2625.

19 Yong M, Kaste M: Association of characteristics of blood pressure profiles and stroke outcomes in the ECASS-II trial. Stroke 2008;39:366-372.

20 Larrue V, von Kummer R, del Zoppo G, Bluhmki E: Hemorrhagic transformation in acute ischemic stroke: potential contributing factors in the European Cooperative Acute Stroke Study. Stroke 1997;28:957-960.

21 Leonardi-Bee J, Bath PM, Phillips SJ, Sandercock PA: Blood pressure and clinical outcomes in the International Stroke Trial. Stroke 2002;33:1315-1320.

22 The NINDS t-PA Stroke Study Group: Intracerebral hemorrhage after intravenous t-PA therapy for ischemic stroke. Stroke 1997;28:2109-2118.

23 IMS Study Investigators: Hemorrhage in the Interventional Management of Stroke study. Stroke 2006;37: 847-851.

24 Levy DE, Brott TG, Haley EC Jr, et al: Factors related to intracranial hematoma formation in patients receiving tissue-type plasminogen activator for acute ischemic stroke. Stroke 1994;25:291-297.

25 Vemmos KN, Tsivgoulis G, Spengos K, et al: Blood pressure course in acute ischaemic stroke in relation to stroke subtype. Blood Press Monit 2004;9:107-114. 